\title{
The role of osteopontin and tumor necrosis factor alpha receptor-1 in xenobiotic-induced cholangitis and biliary fibrosis in mice
}

\author{
Peter Fickert ${ }^{1}$, Andrea Thueringer ${ }^{2}$, Tarek Moustafa ${ }^{1}$, Dagmar Silbert ${ }^{1}$, Judith Gumhold ${ }^{1}$, Oleksiy Tsybrovskyy ${ }^{2}$, \\ Margitta Lebofsky ${ }^{3}$, Hartmut Jaeschke ${ }^{3}$, Helmut Denk ${ }^{2}$ and Michael Trauner $^{1}$
}

Proinflammatory and profibrotic cytokines such as osteopontin (OPN) and tumor necrosis factor-alpha receptor-1 (TNFR 1 ) may be critically involved in the pathogenesis of cholangiopathies and biliary fibrosis. We therefore aimed to determine the role of genetic loss of either OPN or TNFR 1 in 3,5-diethoxycarbonyl-1,4-dihydrocollidine (DDC)-fed mice as a model of xenobiotic-induced sclerosing cholangitis with biliary-type liver fibrosis using respective knock-out mice. OPN and TNFR knock-out mice were fed a 0.1\% DDC-supplemented diet for 4 weeks and compared with corresponding wild-type (WT) controls. Liver morphology (H\&E staining), serum markers of liver injury and cholestasis (ALT, AP, bilirubin), markers of inflammation in liver (CD11b and F4/80 immunostaining, mRNA expression of iNOS, MCP-1, IL-1 $\beta$, INF- $\gamma$, TNF- $\alpha$ and OPN), degree of ductular reaction (immunohistochemistry with morphometric analysis and western blotting for cholangiocyte-specific marker keratin 19) and degree of liver fibrosis (Sirius-red staining, hepatic hydroxyproline content

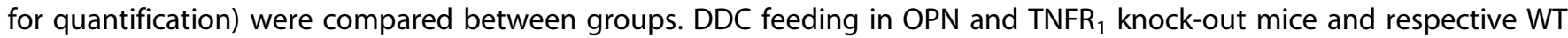
controls resulted in comparable extent of liver injury, inflammatory response, ductular reaction and liver fibrosis. Our data indicate that genetic loss of neither OPN nor TNFR 1 significantly effects on the pathogenesis of DDC-induced sclerosing cholangitis, ductular reaction and resulting biliary fibrosis.

Laboratory Investigation (2010) 90, 844-852; doi:10.1038/labinvest.2010.61; published online 5 April 2010

KEYWORDS: bile acids; cholangiocytes; cholestasis; cytokines; ductular reaction; sclerosing cholangitis

Cholangiopathies such as primary sclerosing cholangitits (PSC), secondary sclerosing cholangitis (SSC), primary biliary cirrhosis and drug-induced bile duct damage may result in a vanishing bile duct syndrome that can progress to biliary cirrhosis; these disorders represent a major indication for liver transplantation and cause of liver-related death. ${ }^{1}$ In spite of the profound differences among cholangiopathies in regard to their pathogenesis, pathological features and clinical presentation, these disorders share several common features, such as (i) bile duct epithelial cells (BECs) represent major victims in cholangiopathies, (ii) the inflammatory infiltrate is focused to portal fields and especially to bile ducts and (iii) ductular reaction as well as (iv) biliary fibrosis with characteristic porto-portal bridging and broadened fibrotic portal fields are frequently observed (for reviews, please see Lazaridis et $a l^{1}, \mathrm{Lu}$ and $\mathrm{Mack}^{2}$, Strazzabosco et $a l^{3}$ and Xia et $\left.a l^{4}\right)$. More specifically, the distinct bile duct lesion in PSC and SSC is characterized by a fibro-obliterative reaction with onion-skin-type periductal fibrosis of the medium sized and large bile ducts. Chronic 3,5-diethoxycarbonyl-1,4-dihydrocollidine (DDC) feeding in mice is a well-established model to study Mallory-Denk body formation, which are hepatocellular inclusion bodies characteristically associated with alcoholic and non-alcoholic steatohepatitis, metabolic liver diseases (eg, Wilson's disease and other forms of copper toxicities) and chronic cholestatic liver diseases. ${ }^{5}$ In addition, we have previously shown that DDC feeding in mice results in cholangitis with pronounced ductular

${ }^{1}$ Laboratory of Experimental and Molecular Hepatology, Division of Gastroenterology and Hepatology, Department of Medicine, Medical University of Graz, Graz, Austria; ${ }^{2}$ Department of Pathology, Medical University of Graz, Graz, Austria and ${ }^{3}$ Department of Pharmacology, Toxicology and Therapeutics, University of Kansas Medical Center, Kansas City, KS, USA

Correspondence: Dr M Trauner, MD, Laboratory of Experimental and Molecular Hepatology, Division of Gastroenterology and Hepatology, Department of Medicine, Medical of University Graz, Auenbruggerplatz 15, Graz A-8036, Austria.

E-mail: michael.trauner@meduni-graz.at

Received 25 September 2009; revised 17 December 2009; accepted 17 December 2009 
reaction, onion-skin-type like periductal fibrosis and finally liver fibrosis of the biliary type. ${ }^{6}$ Therefore, the model of DDC-induced cholangiopathy and biliary fibrosis in mice shares several specific pathological hallmarks with at least some human cholangiopathies associated with biliary type of liver fibrosis, in particular PSC and SSC. This model may thus be useful to investigate the mechanisms of chronic cholangiopathies and their sequels including liver fibrosis of the biliary type.

Specific cytokines may have a pivotal role in the orchestrated interplay between activated BECs, extracellular matrix-producing cells such as hepatic stellate cells and periductal myofibroblasts, inflammatory cells (eg, neutrophils, natural killer cells, Kupffer cells) and vascular smooth muscle cells localized in vessel walls, ultimately causing a cholangiopathy-related biliary fibrosis. ${ }^{7-10}$ Hepatic osteopontin (OPN) expression strongly correlates with ductular reaction and the degree of liver fibrosis in patients with biliary atresia. ${ }^{11}$ In addition, OPN was shown to be critically engaged in the hepatic inflammatory response and fibrogenesis in alcoholic steatohepatitis (ASH) ${ }^{6,12}$ Interestingly enough, expression of OPN in renal tubular epithelial cells is considered a central mediator and marker of epithelial-mesenchymal transition (EMT), a term that refers to the phenotypic conversion of tubular epithelial cells to differentiated matrix-producing cells. The process of EMT has received great attention and its role in kidney fibrosis has been studied extensively in recent years. ${ }^{13,14}$ Moreover, the EMT concept has been successfully translated to liver fibrogenesis. ${ }^{15-21}$ Especially in cholestatic liver diseases and their corresponding animal models EMT, namely from bile duct epithelial cells into myofibroblasts, is supposed to have a pivotal role in the initiation and perpetuation of liver fibrosis. ${ }^{15,17}$ As such, previous studies in the DDC-fed mouse model showed pronounced induction of hepatic OPN in BECs (including BECs in reactive ductules) and hepatocytes in acinar zone 1 according to those lobular regions in which adjacent porto-portal septa develop later in the disease course. ${ }^{6}$ These findings pointed toward a potential pathogenetic role of OPN in DDC-induced biliary type of liver fibrosis. However, the relative contribution of EMT to liver fibrosis is still a matter of active debate and little is known regarding the specific role of OPN in the initiation and progression of liver fibrosis of the biliary type.

Tumor necrosis factor alpha and its receptor-1 $\left(\mathrm{TNFR}_{1}\right)$ represent key molecules critically engaged in the pathogenesis of numerous liver diseases including viral or toxin-induced hepatitis and its sequel liver fibrosis. ${ }^{22-24}$ In addition, antiTNF- $\alpha$ antibodies were shown to reduce hepatic inflammation and fibrosis. ${ }^{25,26}$ We have recently shown induction of TNF- $\alpha$ mRNA expression in Kupffer cells, endothelial cells, and interestingly in reactive BECs in DDC-fed mice ${ }^{6}$ suggesting that activation of TNF receptor pathways may also be critically engaged in the pathogenesis of DDC-induced cholangiopathy and resulting biliary fibrosis. However, the re- lative contribution of $\mathrm{TNFR}_{1}$ activation in the pathobiology of cholangitis and biliary fibrosis in the DDC model remains elusive.

On the basis of these findings and unresolved questions, we hypothesized that $\mathrm{TNFR}_{1}$ and OPN are critically engaged in the pathogenesis of DDC-induced cholangiopathy with biliary fibrosis. This hypothesis was addressed by studying the inflammatory and fibrotic response to DDC in TNFR and OPN knock-out mice in comparison with their corresponding wild-type (WT) controls.

\section{MATERIALS AND METHODS Animals}

Experiments were performed with 2-month-old male mice weighing 25-30 g. OPN (stock \# 004936; congenic C57BL/6) and $\mathrm{TNFR}_{1}$ (stock \# 003242; C57BL/6J) knockout mice (from Jackson Laboratory) and respective WT mice were fed a $0.1 \%$ DDC-supplemented diet for 4 weeks, housed with a 12:12 h light:dark cycle, and permitted water ad libitum. Controls were fed a standard mouse diet (Sniff, Soest, Germany). The experimental protocols were approved by the local Animal Care and Use Committee according to criteria outlined in the Guide for the Care and Use of Laboratory Animals prepared by the National Academy of Sciences, as published by the National Institutes of Health (NIH publication 86-23, revised 1985).

\section{Serum Biochemical Analysis and Liver Histology}

Serum samples were stored at $-70{ }^{\circ} \mathrm{C}$ until analysis of alanine transaminase (ALT) and alkaline phosphatase (AP) and bilirubin by a Hitachi 917 analyzer (Boehringer Mannheim, Mannheim, Germany). For conventional light microscopy, livers were fixed in $4 \%$ neutral buffered formaldehyde solution and embedded in paraffin. Sections $(4 \mu \mathrm{m}$ thick) were stained with $\mathrm{H} \& \mathrm{E}$.

\section{Immunohistochemical Studies}

Antibodies and conditions for immunohistochemical staining of Kupffer cells (F4/80), BECs (keratin 19; K19), inflammatory cells such as neutrophil granulocytes and macrophages (CD11b), vascular cell adhesion molecule (VCAM) and $\alpha$-smooth muscle antigen ( $\alpha$-SMA) are summarized in Supplementary Table 1. For morphometry of K19-positive bile ducts, the measurements were performed using of a semiautomatic system for image analysis. This consisted of a microscope (Nikon E600; Nikon, Osaka, Japan), a video camera (Sony DXC-930P; Sony, Tokyo, Japan), a frame grabber (Integral Tech Intrigue Pro, Indianapolis, IN, USA) and a personal computer with image analysis software (Optimas 6.51; Media Cybernetics Inc, Carlsbad, CA, USA) installed. Approximate borders of a portal field were traced with a mouse by the operator at $\times 20$ objective magnification. A specially developed macro command served for automatic identification and measurement of the total area of cells positive for K19 within the given portal field. In 
addition, the area of the corresponding portal vein was also measured and used in statistical analysis for adjustment for the size of the portal fields. All portal fields within each sample were measured (9-35 portal fields per slide). Obvious artefacts such as unspecific staining of necrotic areas, as well as incompletely cut portal fields at the edges of the specimens, were excluded from analysis.

\section{Measurement of Hepatic Hydroxyproline Content}

Quantification of liver fibrosis was carried out by measurement of hepatic hydroxyproline concentration by a calorimetric method. In brief, mouse liver lobe 3 (left liver lobe) was homogenized in $6 \mathrm{~N} \mathrm{HCl}$ and hydrolyzed overnight at $110^{\circ} \mathrm{C}$. Afterward hydrolysates were filtered, neutralized with $\mathrm{NaOH}$ and oxidized with chloramine-T. This was followed by a reaction with perchloric acid and p-dimethylaminobenzaldehyde resulting in the formation of a chromophore quantified photometrically at $565 \mathrm{~nm}$ wavelength.

\section{Determination of Hepatic mRNA Levels Using Q-PCR}

RNA was extracted and reversely transcribed into cDNA. PCR reaction $(20 \mu \mathrm{l})$ contained $12.5 \mathrm{ng} \mathrm{cDNA}, 330 \mathrm{nM}$ of each primer and $10.5 \mu \mathrm{l}$ of SYBR Green Master mix (Applied Biosystems). Expression levels were normalized to 18sRNA. Primers used are summarized in Supplementary Table 2. In addition, 30 cycles of PCR were performed and $10 \mu \mathrm{l}$ loaded on a $3 \%$ agarose gel to determine the size and specificity of the PCR product. This step describes the validation of primer specificity used for Q-PCR.

\section{Western Blotting}

Antibodies against VCAM, K19, $\beta$-actin and $\alpha$-SMA as well as conditions for western blotting are summarized in Supplementary Table 3. In short, liver homogenates ( $30 \mu \mathrm{g}$ of protein) were run on $10 \%$ sodium dodecyl sulfate polyacrylamide gels, transferred to nitrocellulose and blotted with the respective monoclonal antibodies given in Supplementary Table 3. Binding was detected by using peroxidaseconjugated rabbit immunglobulins against mouse and rat immunglobulins (Dako), and peroxidase activity was visualized by using the enhanced chemiluminescence method (ECL) western blotting detection system.

\section{Statistical Analysis}

Data are reported as arithmetic means \pm s.d. of five animals in each group. Statistical analysis included Student's $t$-test when appropriate or analysis of variance with Bonferroni post-testing when three or more groups were compared, using the Sigmastat statistics (Jandel Scientific, San Rafael, CA, USA). A $P$-value $<0.05$ was considered significant. Morphometric data for K19 represented a hierarchical structure, were portal fields (level 1 observation units) were nested within mice (level 2 observation units). Therefore, a special kind of statistical method, namely multilevel models and corresponding software (MlwinN 1.1) were used. A linear regression model with bootstrap-corrected estimates was fitted to calculate means and confidence intervals for K19-positive areas in each of the study groups and to test the significance of the effects of genotype and diet on the amount of bile ducts. Portal vein areas were included in the model as a baseline parameter to account for the size of the portal fields. To fulfill the normality assumption, bile duct and portal vein areas were logarithmically transformed.

\section{RESULTS}

Genetic Loss of OPN and TNFR 1 has no Major Effect on the Hepatic Inflammatory Response in DDC-Fed Mice

DDC feeding in WT mice led to a pronounced hepatic inflammatory response characterized by a mixed inflammatory infiltrate accentuated in portal fields, especially near bile ducts, with predominating neutrophil granulocytes (shown in Supplementary Figures 1 and 2). This was accompanied by the induction of a reactive phenotype of BECs characterized by overexpression of proinflammatory cyto- and chemokines (eg, TNF- $\alpha$, VCAM, OPN). ${ }^{6}$ In addition, we previously observed substantial induction of cholangiocellular and hepatocellular OPN expression along the margins of the liver acinus representing the lobular region with the highest inflammatory activity. ${ }^{6}$ On the basis of these findings, we hypothesized that this inflammatory response is blunted in $\mathrm{OPN}^{-1-}$ and $\mathrm{TNFR}_{1}^{-1-}$ mice. Immunohistochemistry for the Kupffer cell marker F4/80 revealed similar intensity in response to DDC feeding in all genotypes tested (Figures $1 \mathrm{~b}-\mathrm{d}$ ). The lobular distribution and staining pattern was also comparable. In addition, CD11b immunohistochemistry (staining cells of the monocytic lineage including neutrophil granulocytes but also dendritic cells and NK cells) revealed a comparable staining pattern in response to DDC feeding irrespective of presence or absence of OPN and TNFR 1 (Figures $1 \mathrm{f}-\mathrm{h}$ ). In contrast to the observed even panlobular F4/80 staining pattern, positivity for CD11b was concentrated to portal fields and the margins of the adjacent hepatocytes. We also observed no genotype-specific changes in respect to the composition of the portal infiltrate because neutrophil granulocytes were next to macrophages the predominant cell type also in the studied knock-out strains as observed on H\&E-stained liver sections (shown for TNFR ${ }_{1}^{-1-}$ mice in Supplementary Figure 3). This assumption is also in line with the dissimilar lobular staining pattern in regard to F4/80 and CD11b positivity in DDC-fed mice (Figure 1). Taken together these findings indicated a comparable inflammatory response in all DDC-fed genotypes.

For further quantification of the inflammatory response induction of F4/80, iNOS, IL1- $\beta$ and IFN $\gamma$ mRNA expression was compared and revealed no significant differences between groups (Figure 2). MCP-1 and TNF- $\alpha$ mRNA levels were significantly increased in all genotypes in response to DDC feeding (Figure 2). Interestingly, MCP-1 expression was lower (without reaching statistical significance because of 

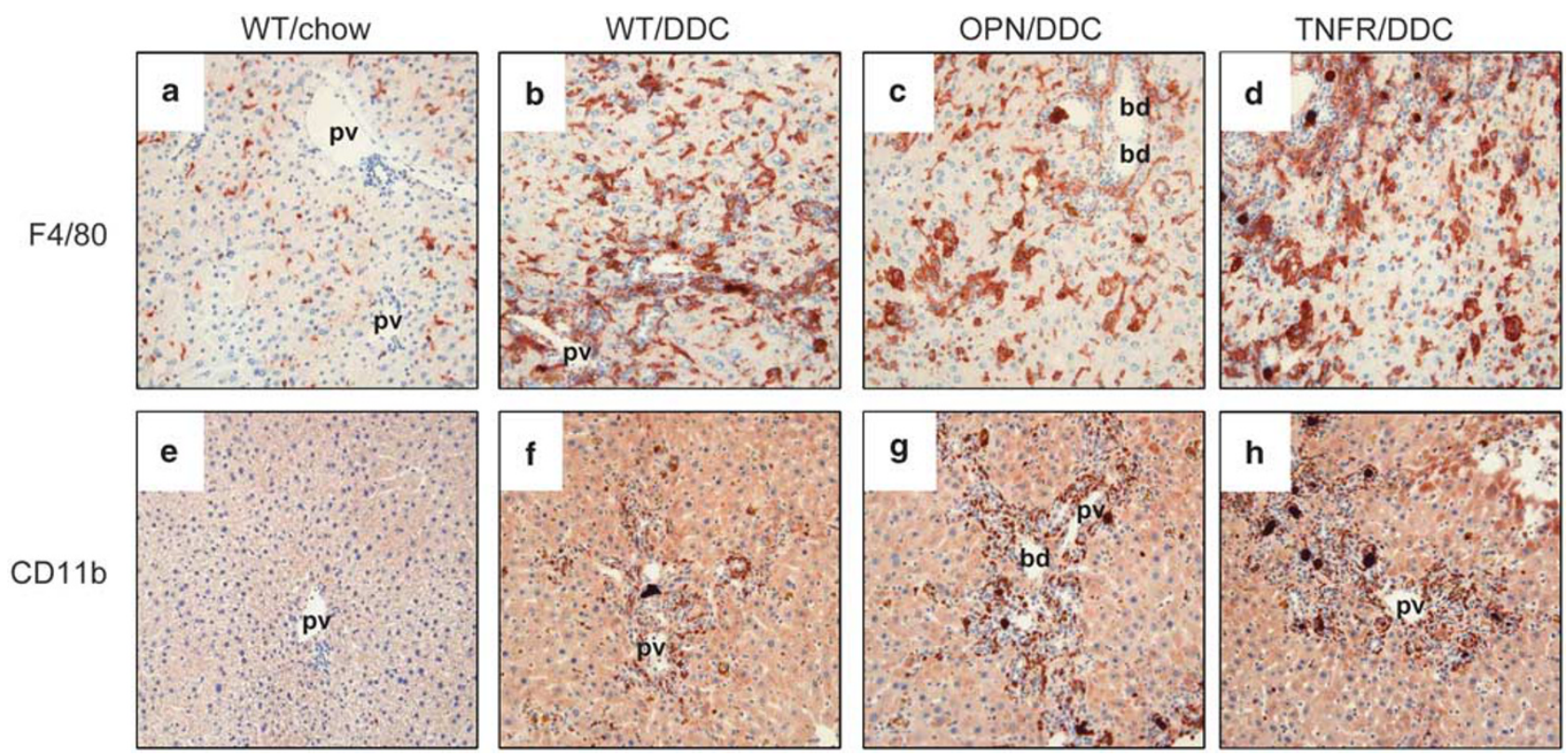

Figure 1 Genetic loss of osteopontin (OPN) and tumor necrosis factor- $\alpha$ receptor-1 (TNFR $)$ has no effect on the composition and density of the inflammatory infiltrate in response to DDC feeding. Immunohistochemistry for F4/80 (a-d) and CD11b (e-h) in chow-fed wild type (WT/chow), WT 4 weeks DDC-fed (WT/DDC), OPN knock-out 4 weeks DDC-fed (OPN/DDC) and TNFR 1 knock-out 4 weeks DDC-fed (TNFR/DDC) mice. (b-d) Please note that in spite of the OPN or TNFR 1 loss 4 weeks DDC-fed knock-out mice show a pronounced hepatic inflammation with no differences in regard to density and lobular distribution of the immunoreactivity. (e-h) In contrast to the observed F4/80 staining pattern the CD11b signal is concentrated and accentuated to portal fields and bile ducts again showing no reduction of the inflammatory response in 4 weeks DDC-fed knock-out mice indicating a comparable inflammatory response compared with WT controls. pv, portal vein; bd, bile duct. Original magnification $\times 20$.

Table 1 ALT and AP serum levels under various experimental conditions

\begin{tabular}{lccc}
\hline $\begin{array}{l}\text { Experimental } \\
\text { group }\end{array}$ & ALT (U/l) & AP $(\mathrm{U} / \mathrm{l})$ & $\begin{array}{c}\text { Bilirubin } \\
\text { (mg per 100 ml) }\end{array}$ \\
\hline WT/chow & $31 \pm 7$ & $85 \pm 14$ & $0.11 \pm 0.06$ \\
WT/DDC & $1271 \pm 521^{*}$ & $2559 \pm 834^{*}$ & $22.59 \pm 4.04^{*}$ \\
OPN/chow & $23 \pm 3$ & $113 \pm 7$ & $0.13 \pm 0.12$ \\
OPN/DDC & $1093 \pm 312^{*}$ & $2456 \pm 474^{*}$ & $16.26 \pm 2.77^{*}$ \\
TNFR/DDC & $1046 \pm 200^{*}$ & $2130 \pm 177^{*}$ & $14.25 \pm 1.20^{*}$ \\
\hline
\end{tabular}

$n=5$ animals in each group; ${ }^{*}$ Indicates $P<0.05$ chow-fed wild-type (WT) animals vs DDC-fed WT, osteopontin knock-out mice (OPN) and tumor necrosis factor alpha receptor-1 (TNFR 1 ) knock out mice; " indicates $P<0.05$ DDC-fed wild-type (WT) animals vs DDC-fed TNFR ${ }_{1}$ knock out mice.

high s.d. in DDC-fed mice) and TNF- $\alpha$ expression was significantly reduced in $\mathrm{TNFR}_{1}^{-1-}$ mice when compared with respective WT controls (Figure 2), suggesting an at least in part reduced inflammatory response in this genotype. Induction of inflammatory genes was comparable between $\mathrm{OPN}^{-1-}$ mice and respective $\mathrm{WT}$ controls in response to DDC apart from the expected undetectable OPN mRNA levels in $\mathrm{OPN}^{-1-}$ mice (Figure 2). We next compared hepatic VCAM expression as a parameter for the hepatic inflammatory response in our model system because induc- tion of VCAM expression in response to DDC feeding is robust and parallels the development of the inflammatory infiltrate. ${ }^{6}$ In contrast to chow-fed controls, VCAM expression was significantly induced in reactive BECs, endothelial cells and Kupffer cells in DDC-fed mice and we again observed no differences in the staining pattern between genotypes (Figures 3b-d). Western blotting to quantify VCAM protein levels (Figure 3) confirmed these findings showing no significant differences between DDC-fed $\mathrm{OPN}^{-1-}, \mathrm{TNFR}_{1}^{-1-}$ mice and their respective WT controls. Thus, in spite of some differences in the induction of proinflammatory genes (ie, TNF- $\alpha$ and MCP-1 mRNA expression levels in DDC-fed $\mathrm{TNFR}_{1}^{-1-}$ mice) these findings suggest that a single genetic loss of OPN or $\mathrm{TNFR}_{1}$ signalling has no major effect on the inflammatory response in DDC-fed mice.

\section{Genetic Loss of OPN and TNFR 1 has no Effect on the Degree of Ductular Reaction and Biliary Fibrosis in DDC- Fed Mice}

Previous studies revealed substantial overexpression of OPN and TNF- $\alpha$ in BECs of reactive ductules in the DDC model, ${ }^{6}$ leading us to hypothesize that these molecules may be critically engaged in the ductular reaction typically associated with liver fibrosis of the biliary type. More specifically, induction of OPN in DDC-fed mice was preferentially observed in hepatocytes along the margins of the liver acinus wherein porto-portal septa are formed later on, ${ }^{6}$ suggesting a 


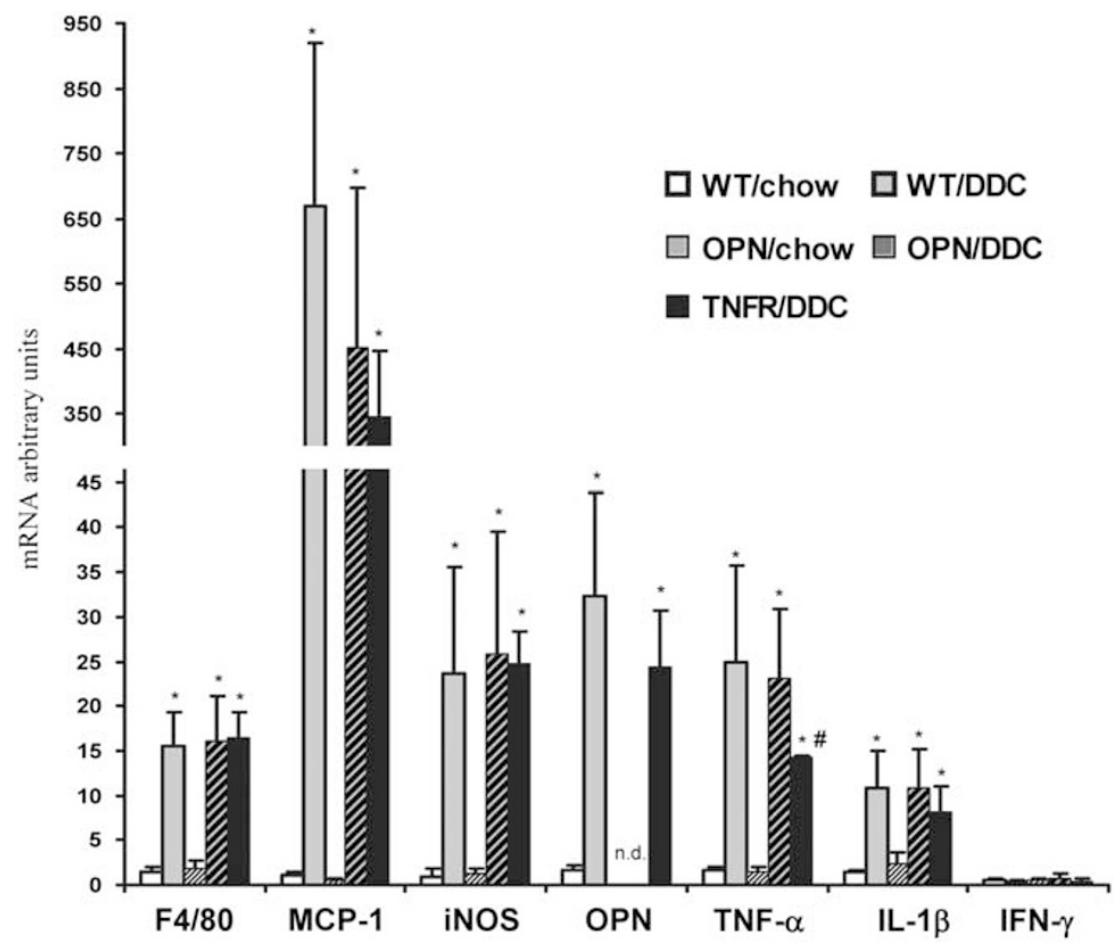

Figure 2 Genetic loss of osteopontin (OPN) and tumor necrosis factor- $\alpha$ receptor-1 (TNFR 1 ) has no impact on hepatic F4/80 and iNOS mRNA expression. PCR for quantification of hepatic F4/80 and iNOS mRNA levels in chow-fed wild type (WT/chow), WT DDC-fed (WT/DDC), OPN knock-out DDC-fed (OPN/DDC), and TNFR 1 knock-out DDC-fed (TNFR/DDC) mice using SYBR Green. Please note that despite OPN and TNFR loss, DDC-fed mice show comparable expression levels. $n=5$ in each group; ${ }^{*} P<0.05$ chow-fed vs DDC-fed mice.

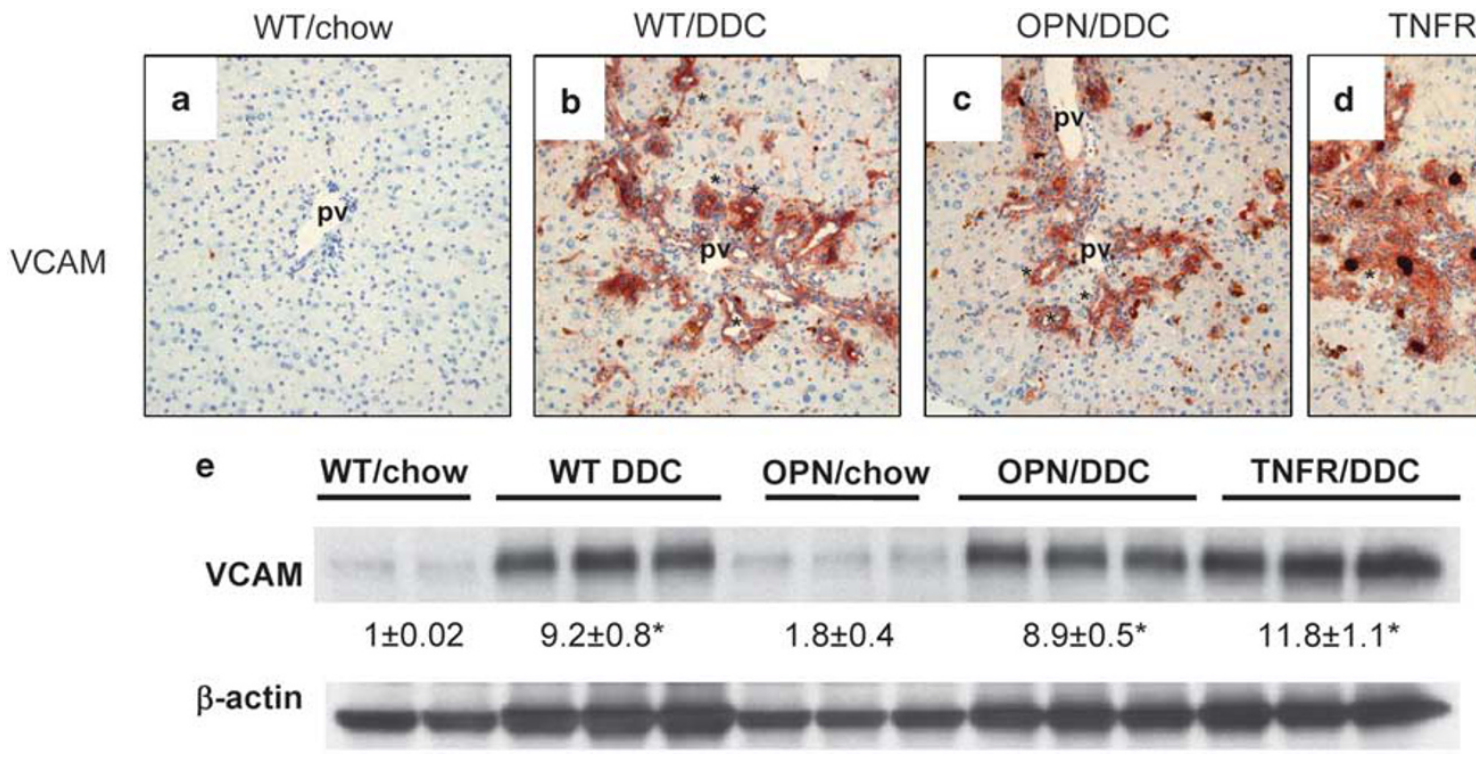

Figure 3 Genetic loss of osteopontin (OPN) and tumor necrosis factor- $\alpha$ receptor- 1 (TNFR 1 ) has no effect on hepatic expression of vascular cell adhesion molecule (VCAM) in response to DDC feeding. Immunohistochemistry for VCAM (a-d) and VCAM protein measurement by western blotting

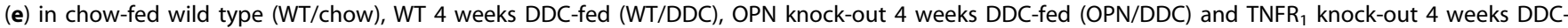
fed (TNFR/DDC) mice. (b-d) Please note that in spite of the OPN and TNFR 1 loss, DDC-fed mice show a comparable hepatic VCAM induction most pronounced in reactive bile ductules (indicated by asterisks), endothelial cells, and Kupffer cells. (e) Hepatic VCAM protein quantification by western blotting in 4 weeks DDC-fed mice revealed significant induction in all genotypes. ${ }^{*}$ Indicates $P<0.05$ chow-fed mice vs DDC-fed mice. pv, portal vein. Original magnification $\times 20$.

possible direct role for OPN in the development of biliary fibrosis. We therefore compared the degree of ductular reaction immunohistochemically and quantified ductular mass by morphometric analysis and western blotting using the cholangiocyte-specific marker K19. Immunohistochemistry for K19 revealed a comparable amount of ductular reaction 

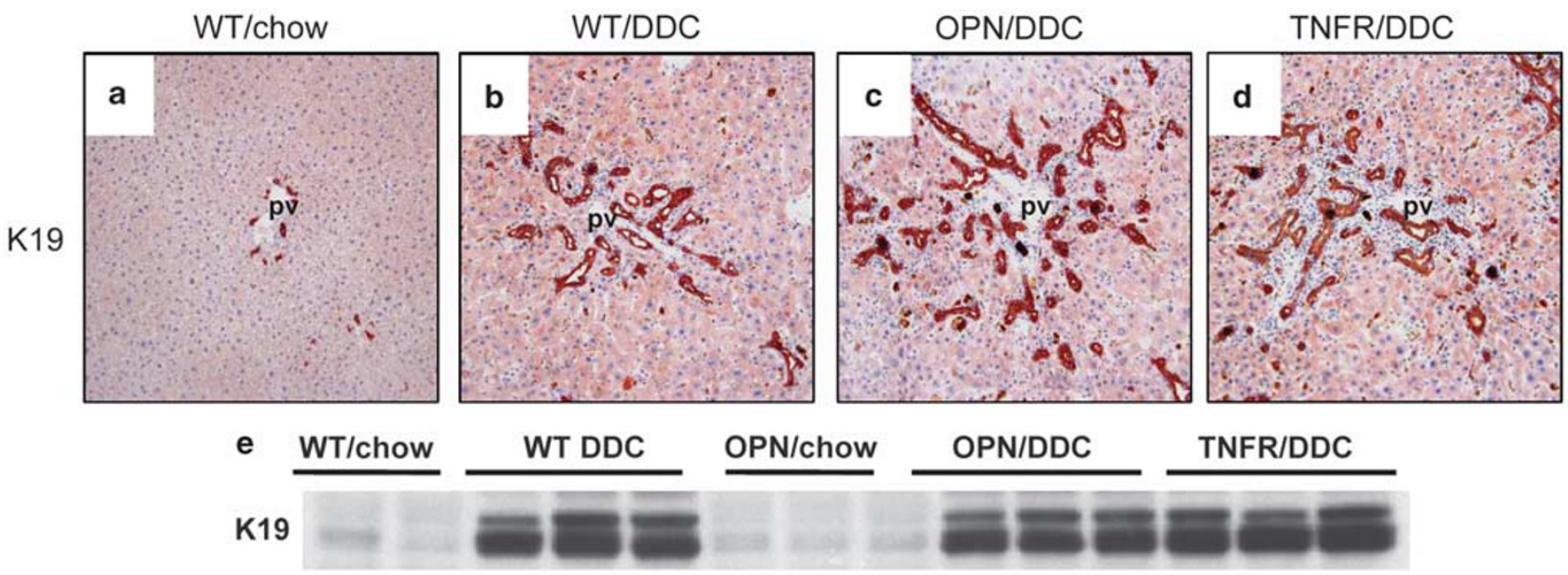

$1 \pm 0.2$

$5.8 \pm 0.6^{*}$

$0.9 \pm 0.07$

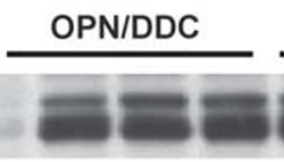

TNFR/DDC

Figure 4 Genetic loss of osteopontin (OPN) and tumor necrosis factor- $\alpha$ receptor $\left(\mathrm{TNFR}_{1}\right)$ has no effect on the degree of ductular reaction in response to DDC-feeding. Immunohistochemistry of keratin 19 (K19) (a-d) and K19 protein measurement by western blotting (e) in chow-fed wild type (WT/chow), WT 4 weeks DDC-fed (WT/DDC), OPN knock-out 4 weeks DDC-fed (OPN/DDC) and TNFR 1 knock-out 4 weeks DDC-fed (TNFR/DDC) mice. (b-d) Please note that OPN and TNFR 1 loss do not reduce ductular reaction in 4 weeks DDC-fed mice. (e) Hepatic K19 protein quantification by western blotting in 4 weeks DDC-fed mice revealed significant and comparable induction in all genotypes. ${ }^{*}$ Indicates $P<0.05$ chow-fed mice $v s$ DDC-fed mice. pv, portal vein. Original magnification $\times 20$.
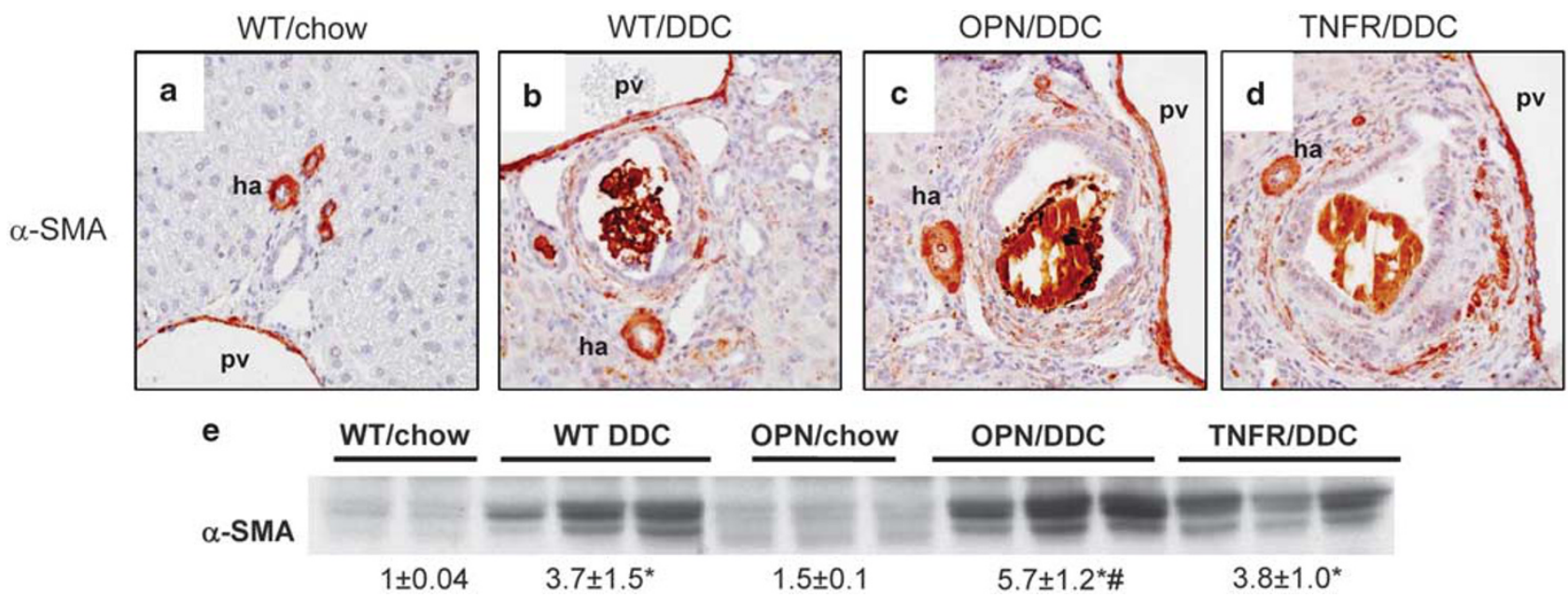

$1 \pm 0.04$

$1.5 \pm 0.1$

\section{$5.7 \pm 1.2^{*} \#$}

$3.8 \pm 1.0^{*}$

Figure 5 Effect of osteopontin (OPN) and tumor necrosis factor- $\alpha$ receptor (TNFR ${ }_{1}$ ) deficiency on the degree of $\alpha$-SMA expression in DDC-fed mice. Immunohistochemistry for $\alpha$-SMA (a-d) and $\alpha$-SMA protein measurement by western blotting (e) in chow-fed wild type (WT/chow), WT 4 weeks DDC-fed (WT/DDC), OPN knock-out 4 weeks DDC-fed (OPN/DDC) and TNFR 1 knock-out 4 weeks DDC-fed (TNFR/DDC) mice. (b-d) Please note that OPN and $\mathrm{TNFR}_{1}$ loss do not reduce hepatic $\alpha$-SMA expression in 4 weeks DDC-fed mice. (e) Hepatic $\alpha$-SMA protein quantification by western blotting in 4 weeks DDC-fed mice revealed significant induction in all genotypes, which was more pronounced in DDC-fed OPN knock-out mice. ${ }^{*}$ Indicates $P<0.05$ chow-fed mice vs DDC-fed mice. "Indicates $P<0.05$ DDC-fed OPN knock-out mice vs DDC-fed WT and DDC-fed TNFR 1 knock-out mice. Ha, hepatic artery; pv, portal vein. Original magnification $\times 20$.

in all genotypes tested (Figures $4 \mathrm{~b}-\mathrm{d}$ ). The estimated bile duct area as revealed by morphometric analysis showed significant increased bile duct area in all DDC-fed genotypes compared with chow-fed controls (chow-fed WT $1.9 \pm 0.3$; DDC-fed WT $4.8 \pm 0.3$; DDC-fed $\mathrm{OPN}^{-l-} 4.3 \pm 0.4$; DDCfed $\mathrm{TNFR}_{1}^{-1-} 5.0 \pm 0.3$; differences between chow-fed and DDC-fed groups statistically significant). However, the in- crease in bile duct area in the different genotypes in response to DDC was similar. These data were further confirmed by similar hepatic K19 protein levels (Figure 4e). The finding of comparable hepatic ductular mass in all genotypes stressed with DDC-supplemented diet indicates that single genetic loss of OPN or TNFR ${ }_{1}$ has no major direct effect on ductular reaction in this model. This assumption was further 


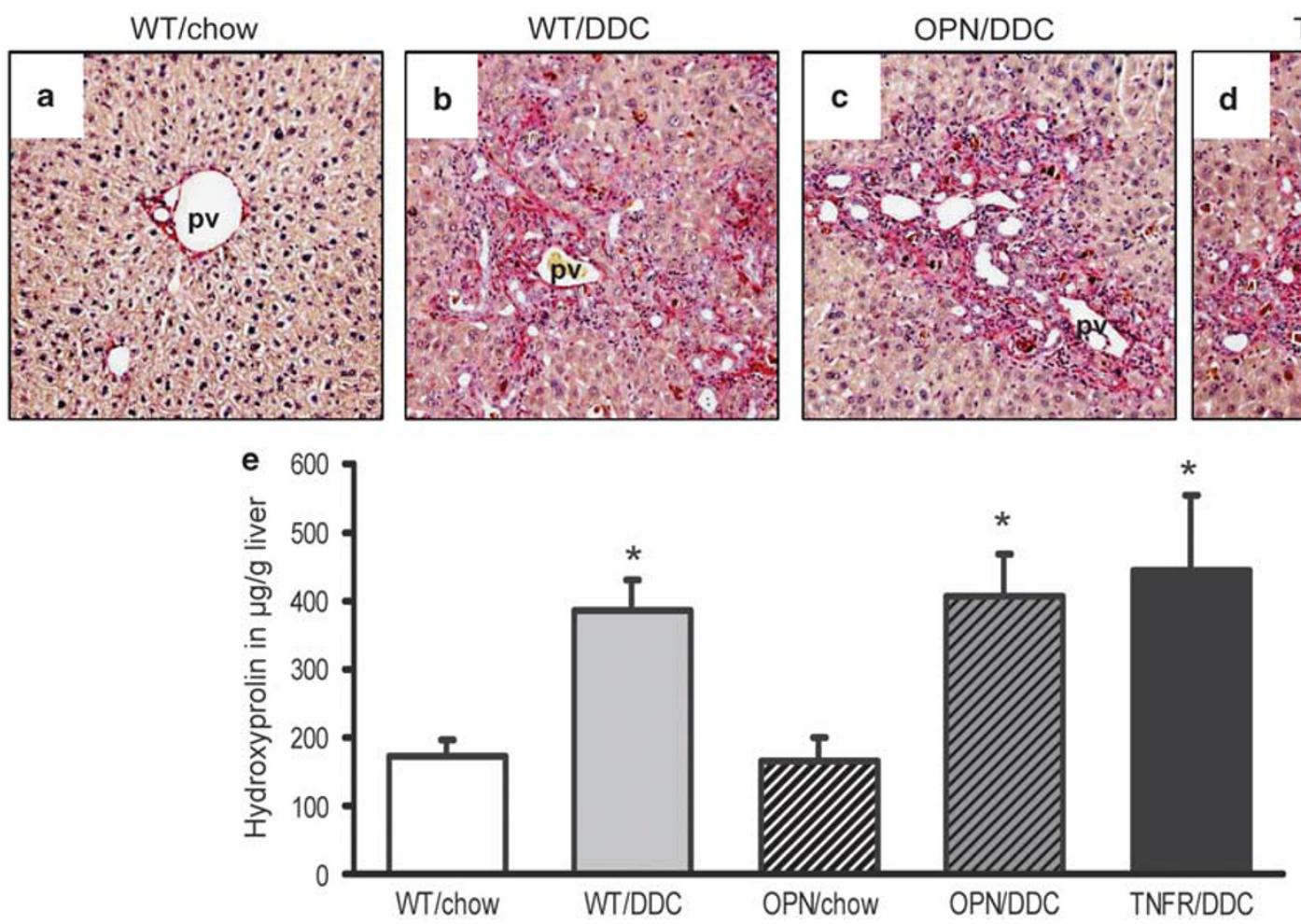

Figure 6 Genetic loss of osteopontin (OPN) and tumor necrosis factor- $\alpha$ receptor- 1 (TNFR 1 ) does not decrease the degree of biliary fibrosis in DDC-fed mice. Sirius-red staining (a-d) and hepatic hydroxyproline measurement (e) in chow-fed wild type (WT/chow), WT 4 weeks DDC-fed (WT/DDC), OPN knock-out 4 weeks DDC-fed (OPN/DDC), and TNFR 1 knock-out 4 weeks DDC-fed (TNFR/DDC) mice. (b-d) Sirius-red staining showed comparable staining pattern in all DDC-fed genotypes. (e) Biochemical quantification of liver fibrosis by hepatic hydroxyproline measurement showing that genetic loss of OPN or TNFR ${ }_{1}$ does not reduce the degree of biliary fibrosis in DDC-fed mice. pv, portal vein. ${ }^{*}$ Indicates $P<0.05$ chow-fed mice $v s$ DDC-fed mice. Original magnification $\times 20$.

supported by a comparable proliferative response in all DDCtreated genotypes as revealed by immunohistochemistry using the proliferation marker Ki-67 (data not shown).

Biliary fibrosis in DDC-fed mice is accompanied by proliferation and activation of periductal myofibroblasts of medium-sized and large bile ducts, which typically goes hand in hand with induced $\alpha$-SMA expression. ${ }^{6}$ Comparing immunohistochemistry for $\alpha$-SMA revealed no differences between DDC-fed genotypes (Figures $5 b-d$ ). Hepatic $\alpha$-SMA protein levels were equal between DDC-fed WT and $\mathrm{TNFR}_{1}^{-1-}$ mice (Figures $5 \mathrm{~b}-\mathrm{d}$ ). Induction of $\alpha$-SMA protein expression, however, was even more pronounced in DDC-fed $\mathrm{OPN}^{-1-}$ mice compared with DDC-fed $\mathrm{TNFR}_{1}^{-1-}$ mice (Figure 5e). These findings suggest that $\mathrm{OPN}$ and $\mathrm{TNFR}_{1}$ signalling may not have a pivotal role for the induction of the proliferative response and activation of periductal myofibroblasts in the DDC model. In addition, Sirius-red stain and hepatic hydroxyproline levels also failed to reveal any significant differences between DDC-fed genotypes (Figure 6). Taken together, the finding of a comparable fibrotic response in all DDC-fed genotypes make a direct or unique role of OPN or TNFR ${ }_{1}$ for the pathogenesis of biliary fibrosis in this model rather unlikely. Assessment of liver injury on H\&Estained liver sections and by serum ALT also revealed no significant differences between DDC-fed knockout mice and respective WT controls (Table 1). In addition, we found no significant differences in regard to serum AP levels indicating a comparable degree of cholestasis (Table 1). Interestingly, $\mathrm{TNFR}_{1}^{-1-}$ mice showed significantly lower serum bilirubin levels in response to DDC suggesting a decreased susceptibility of this genotype to cholestasis in this model (Table 1). It is tempting to hypothesize that this finding could be linked to differences in canalicular Mrp2 expression (known to be cytokine sensitive), ${ }^{27}$ which deserves further investigations. Together these findings suggest that genetic loss of OPN or $\mathrm{TNFR}_{1}$ has no major effect on the toxic response and defense as well as the degree of cholestasis in DDC-fed mice.

\section{DISCUSSION}

Proinflammatory cytokines and their receptors may be critically engaged in the pathobiology of several cholangiopathies ${ }^{28-30}$ and these molecules may represent attractive therapeutic targets. ${ }^{3,31}$ We therefore aimed to model the specific effects of genetic OPN and TNFR 1 loss in DDC-fed mice with sclerosing cholangitis and biliary type of liver fibrosis, as this cholangiopathy model was previously shown to share several pathogenetic and morphological hallmarks of sclerosing cholangitis in humans and is associated with pronounced induction and activation of these inflammatory pathways. ${ }^{6}$ Taken together, our data 
largely dismiss a direct and pivotal role for OPN and $\mathrm{TNFR}_{1}$ signalling for the initiation and perpetuation of biliary fibrosis in a mouse model of xenobiotic-induced liver injury.

Proinflammatory cytokines and chemokines and their corresponding receptors have a critical role in the initiation and perpetuation of various types of liver fibrosis. ${ }^{32}$ As such, the potential role of OPN in the pathogenesis of liver fibrosis gained substantial interest and is currently under debate. ${ }^{11,12,33-37}$ We have previously shown pronounced induction of hepatic OPN expression in DDC-fed mice and immunohistochemical studies have revealed that OPN is primarily expressed in BECs (including BECs in reactive ductules) and hepatocytes in acinar zone $1 .^{6}$ In addition, OPN staining was most pronounced in periportal lobular regions in which adjacent porto-portal septa develop in DDC-fed mice. ${ }^{6}$ OPN expression levels were shown to reflect the disease activity of sclerosing cholangitis in Abcb4 (Mdr2) knockout mice. ${ }^{38}$ These findings led us to hypothesize that the observed DDC-induced OPN expression may represent a main trigger for the pronounced ductular reaction and biliary fibrosis in DDC-fed mice. In addition, these findings could indicate a potential role of OPN-induced EMT in this model. The role of OPN in EMT has raised considerable interest, especially in regard to its effect on kidney fibrosis. ${ }^{13,14}$ In addition, the concept of EMT has recently also gained great attention in the pathogenesis of liver fibrosis. ${ }^{15-21}$ Together these findings led us to hypothesize that OPN may be critically involved in ductular proliferation and fibrosis of the biliary type. The findings of this study clearly show, however, that genetic loss of OPN has no major effect on the degree of ductular reaction and liver fibrosis in DDC-fed mice and in consequence, we also dismiss a central pathogenetic role for OPN-induced EMT as a major cause for biliary fibrosis in the applied model system.

However, several important and critical aspects, including limitations of the used mouse models, have to be taken into account. As such, gene knock-out technology in mice frequently involves the risk of adaptive and compensatory processes. Probably even more important in the case of OPN, cytokines habitually act within redundant networks and pathways. Our experimental findings obtained in the DDC-fed mouse model, therefore, do not allow the direct conclusion that OPN has no role in biliary fibrosis induced under different experimental conditions. An additional question is the unknown trigger for the induction of cholangiocelluar OPN expression in DDC-fed mice. Candidates include potentially toxic bile acids (eg, cholic acid) or a biliary excreted DDC metabolite. However, experiments using isolated BECs failed to show induced OPN expression in response to bile acid treatment (unpublished observation). Alternatively increased biliary pressure, which is a welldefined trigger for ductular proliferation, ${ }^{39-42}$ may be causative because shear stress was shown to induce OPN expression in various cell types including osteoblasts, ${ }^{43}$ periodontal cells, ${ }^{44}$ aortic smooth muscle cells ${ }^{45}$ and podocytes. ${ }^{46}$ Taken together, the findings of these interesting studies identified OPN as a stretch-adapting molecule in various cell types, which may also be the case for BECs under cholestatic conditions especially with an obstructive component.

TNF- $\alpha$ and its receptors are key molecules in the hepatic inflammatory response, execution of apoptosis and regulation of liver regeneration. TNF- $\alpha$ may be critically involved especially in ASH/NASH pathobiology and several current therapeutic strategies (eg, statin treatment, neutralizing antibodies) are aiming at modulation of this critical signalling pathway. In addition, members of the TNF superfamily may represent major players in the immunobiology of sclerosing cholangitis and associated biliary fibrosis ${ }^{30,47}$ as such infiltrating lymphocytes could attack and destroy BECs via TNFR signalling. Moreover, increased levels of TNF- $\alpha$ may contribute to the oxidative damage to the biliary system. We have previously shown profound induction of TNF- $\alpha$ mRNA, predominantly in portal fields with ductular reaction and in Kupffer cells using in situ hybridization. ${ }^{6}$ TNF- $\alpha$ was previously shown to induce hepatic VCAM expression in a murine endotoxic shock model. ${ }^{48}$ In addition, hepatic VCAM expression was also significantly induced in DDC-fed mice. ${ }^{6}$ We therefore hypothesized that TNF- $\alpha$ and its receptor pathways could be critically involved in the hepatic inflammatory response to DDC (eg, induction of VCAM expression) and consequently the pathobiology of cholangitis and biliary fibrosis in this model. The findings of this study, however, speak against a pivotal role for these molecules. Interestingly, our findings are also in line with the lack of a clinical benefit of anti-TNF strategies such as etanercept and pentoxyfilline in the treatment of cholangiopathies, for example, PSC. ${ }^{49,50}$ Again our data need to be interpreted with caution, as our findings cannot dismiss a role for $\mathrm{TNFR}_{2}$-mediated pathways or alternative activation in DDC-fed $\mathrm{TNFR}_{1}^{-1-}$ mice. In addition, signalling via alternative TNF receptors was sufficient to induced cholangitis in chronic Cyryptosporidium parvum-infected mice in single TNFR knock-out mice. ${ }^{51}$ Alternative strategies to overcome inherent problems of these systems could lay in the generation of conditional knock-out mice.

Nevertheless, on the basis of the findings of this study, we conclude that previously observed OPN and TNF- $\alpha$ overexpression in DDC-fed mice may be more a consequence rather than the cause of the development of sclerosing cholangitis, ductular reaction and biliary fibrosis in DDC-fed mice. In addition, data presented put into question the concept that OPN and $\mathrm{TNFR}_{1}$ are critical to initiate or perpetuate liver fibrosis of the biliary type.

Supplementary Information accompanies the paper on the Laboratory Investigation website (http://www.laboratoryinvestigation.org)

\section{ACKNOWLEDGEMENTS}

We gratefully acknowledge Dr W Erwa (Graz) and colleagues for performing the biochemical analysis of serum liver parameters. This work was supported by grants P-18613 and 19118 to MT from the Austrian Science Foundation and a GEN-AU project grant from the Austrian Ministry for Science to MT, and the National Institutes of Health grant R01 AA12916 to HJ. 


\section{DISCLOSURE/CONFLICT OF INTEREST}

The authors declare no conflict of interest.

1. Lazaridis KN, Strazzabosco M, LaRusso NF. The cholangiopathies: disorders of biliary epithelia. Gastroenterology 2004;127:1565-1577.

2. Lu BR, Mack CL. Inflammation and biliary tract injury. Curr Opin Gastroenterol 2009;25:260-264.

3. Strazzabosco M, Fabris L, Spirli C. Pathophysiology of cholangiopathies. J Clin Gastroenterol 2005;39(4 Suppl 2):S90-S102.

4. Xia X, Demorrow $\mathrm{S}$, Francis $\mathrm{H}$, et al. Cholangiocyte injury and ductopenic syndromes. Semin Liver Dis 2007;27:401-412.

5. Zatloukal K, Stumptner C, Fuchsbichler A, et al. The keratin cytoskeleton in liver diseases. J Pathol 2004;204:367-376.

6. Fickert $P$, Stoger U, Fuchsbichler A, et al. A new xenobiotic-induced mouse model of sclerosing cholangitis and biliary fibrosis. Am J Pathol 2007;171:525-536.

7. Friedman SL. Mechanisms of hepatic fibrogenesis. Gastroenterology 2008;134:1655-1669.

8. Notas G, Kisseleva T, Brenner D. NK and NKT cells in liver injury and fibrosis. Clin Immunol 2009;130:16-26.

9. Parola M, Marra F, Pinzani M. Myofibroblast-like cells and liver fibrogenesis: emerging concepts in a rapidly moving scenario. Mol Aspects Med 2008;29:58-66.

10. Ramadori G, Saile B. Portal tract fibrogenesis in the liver. Lab Invest 2004:84:153-159.

11. Whitington PF, Malladi $P$, Melin-Aldana $H$, et al. Expression of osteopontin correlates with portal biliary proliferation and fibrosis in biliary atresia. Pediatr Res 2005;57:837-844.

12. Apte UM, Banerjee A, McRee R, et al. Role of osteopontin in hepatic neutrophil infiltration during alcoholic steatohepatitis. Toxicol Appl Pharmacol 2005;207:25-38.

13. Zeisberg $M$, Kalluri R. The role of epithelial-to-mesenchymal transition in renal fibrosis. J Mol Med 2004;82:175-181.

14. Zeisberg M, Neilson EG. Biomarkers for epithelial-mesenchymal transitions. J Clin Invest 2009;119:1429-1437.

15. Demetris AJ, Specht S, Nozaki I, et al. Small proline-rich proteins (SPRR) function as $\mathrm{SH} 3$ domain ligands, increase resistance to injury and are associated with epithelial-mesenchymal transition (EMT) in cholangiocytes. J Hepatol 2008;48:276-288.

16. Omenetti A, Porrello A, Jung $Y$, et al. Hedgehog signaling regulates epithelial-mesenchymal transition during biliary fibrosis in rodents and humans. J Clin Invest 2008;118:3331-3342.

17. Robertson H, Kirby JA, Yip WW, et al. Biliary epithelial-mesenchymal transition in posttransplantation recurrence of primary biliary cirrhosis Hepatology 2007;45:977-981.

18. Rygiel KA, Robertson $\mathrm{H}$, Marshall $\mathrm{HL}$, et al. Epithelial-mesenchymal transition contributes to portal tract fibrogenesis during human chronic liver disease. Lab Invest 2008;88:112-123.

19. Syn WK, Jung Y, Omenetti A, et al. Hedgehog-mediated epithelial-tomesenchymal transition and fibrogenic repair in nonalcoholic fatty liver disease. Gastroenterology 2009;137:1478-1488.

20. Yue HY, Yin C, Hou JL, et al. Hepatocyte nuclear factor 4\{alpha\} attenuates hepatic fibrosis in rats. Gut 2010;59:236-246.

21. Zeisberg $M$, Yang $C$, Martino $M$, et al. Fibroblasts derive from hepatocytes in liver fibrosis via epithelial to mesenchymal transition. J Biol Chem 2007;282:23337-23347.

22. Polyzos SA, Kountouras J, Zavos C. Nonalcoholic fatty liver disease: the pathogenetic roles of insulin resistance and adipocytokines. Curr Mol Med 2009;9:299-314.

23. Rutherford A, Chung RT. Acute liver failure: mechanisms of hepatocyte injury and regeneration. Semin Liver Dis 2008;28:167-174.

24. Sheikh MY, Choi J, Qadri I, et al. Hepatitis C virus infection: molecular pathways to metabolic syndrome. Hepatology 2008;47:2127-2133.

25. Bahcecioglu IH, Koca SS, Poyrazoglu OK, et al. Hepatoprotective effect of infliximab, an anti-TNF-alpha agent, on carbon tetrachlorideinduced hepatic fibrosis. Inflammation 2008;31:215-221.

26. Koca SS, Bahcecioglu IH, Poyrazoglu OK, et al. The treatment with antibody of TNF-alpha reduces the inflammation, necrosis and fibrosis in the non-alcoholic steatohepatitis induced by methionine- and choline-deficient diet. Inflammation 2008;31:91-98.

27. Trauner $M$, Arrese $M$, Soroka $C J$, et al. The rat canalicular conjugate export pump (Mrp2) is down-regulated in intrahepatic and obstructive cholestasis. Gastroenterology 1997;113:255-264.
28. Aron $\mathrm{JH}$, Bowlus $\mathrm{CL}$. The immunobiology of primary sclerosing cholangitis. Semin Immunopathol 2009;31:383-397.

29. O'Mahony CA, Vierling JM. Etiopathogenesis of primary sclerosing cholangitis. Semin Liver Dis 2006;26:3-21.

30. Aoki CA, Bowlus CL, Gershwin ME. The immunobiology of primary sclerosing cholangitis. Autoimmun Rev 2005;4:137-143.

31. Alvaro D, Mancino MG. New insights on the molecular and cell biology of human cholangiopathies. Mol Aspects Med 2008;29:50-57.

32. Bataller R, Brenner DA. Liver fibrosis. J Clin Invest 2005;115: 209-218.

33. Lorena D, Darby IA, Gadeau AP, et al. Osteopontin expression in normal and fibrotic liver. Altered liver healing in osteopontin-deficient mice. J Hepatol 2006;44:383-390.

34. Zhao L, Li T, Wang $\mathrm{Y}$, et al. Elevated plasma osteopontin level is predictive of cirrhosis in patients with hepatitis B infection. Int J Clin Pract 2008;62:1056-1062.

35. Sahai A, Malladi $P$, Melin-Aldana $H$, et al. Upregulation of osteopontin expression is involved in the development of nonalcoholic steatohepatitis in a dietary murine model. Am J Physiol Gastrointest Liver Physiol 2004;287:G264-G273.

36. Sahai $A$, Malladi $P, P a n X$, et al. Obese and diabetic $\mathrm{db} / \mathrm{db}$ mice develop marked liver fibrosis in a model of nonalcoholic steatohepatitis: role of short-form leptin receptors and osteopontin. Am J Physiol Gastrointest Liver Physiol 2004;287:G1035-G1043.

37. Harada K, Ozaki S, Sudo Y, et al. Osteopontin is involved in the formation of epithelioid granuloma and bile duct injury in primary biliary cirrhosis. Pathol Int 2003;53:8-17.

38. Nakken KE, Nygard S, Haaland TK, et al. Gene expression profiles reflect sclerosing cholangitis activity in abcb4 (-/-) mice. Scand J Gastroenterol 2009;44:211-218.

39. Fickert $\mathrm{P}$, Zollner G, Fuchsbichler A, et al. Ursodeoxycholic acid aggravates bile infarcts in bile duct-ligated and Mdr2 knockout mice via disruption of cholangioles. Gastroenterology 2002;123: 1238-1251.

40. Slott PA, Liu MH, Tavoloni N. Origin, pattern, and mechanism of bile duct proliferation following biliary obstruction in the rat. Gastroenterology 1990;99:466-477.

41. Wagner M, Fickert $P$, Zollner $G$, et al. Role of farnesoid X receptor in determining hepatic $A B C$ transporter expression and liver injury in bile duct-ligated mice. Gastroenterology 2003;125:825-838.

42. Wiener SM, Hoyt Jr RF, Deleonardis JR, et al. Manometric changes during retrograde biliary infusion in mice. Am J Physiol Gastrointest Liver Physiol 2000;279:G49-G66.

43. You J, Reilly GC, Zhen X, et al. Osteopontin gene regulation by oscillatory fluid flow via intracellular calcium mobilization and activation of mitogen-activated protein kinase in MC3T3-E1 osteoblasts. J Biol Chem 2001;276:13365-13371.

44. Wongkhantee $S$, Yongchaitrakul T, Pavasant P. Mechanical stress induces osteopontin via ATP/P2Y1 in periodontal cells. J Dent Res 2008;87:564-568.

45. lizuka K, Murakami T, Kawaguchi H. Pure atmospheric pressure promotes an expression of osteopontin in human aortic smooth muscle cells. Biochem Biophys Res Commun 2001;283:493-498.

46. Endlich N, Sunohara M, Nietfeld W, et al. Analysis of differential gene expression in stretched podocytes: osteopontin enhances adaptation of podocytes to mechanical stress. FASEB J 2002;16: 1850-1852.

47. Adams DH, Afford SC. The role of cholangiocytes in the development of chronic inflammatory liver disease. Front Biosci 2002;7:e276-e285.

48. Essani NA, Bajt ML, Farhood A, et al. Transcriptional activation of vascular cell adhesion molecule-1 gene in vivo and its role in the pathophysiology of neutrophil-induced liver injury in murine endotoxin shock. J Immunol 1997;158:5941-5948.

49. Barrie A, Regueiro M. Biologic therapy in the management of extraintestinal manifestations of inflammatory bowel disease. Inflamm Bowel Dis 2007;13:1424-1429.

50. Silveira MG, Lindor KD. Clinical features and management of primary sclerosing cholangitis. World J Gastroenterol 2008;14: 3338-3349.

51. Ponnuraj EM, Hayward AR. Requirement for TNF-Tnfrsf1 signalling for sclerosing cholangitis in mice chronically infected by Cryptosporidium parvum. Clin Exp Immunol 2002;128:416-420. 